\title{
The concept of entropy. Relation between action and entropy
}

\author{
J.-P.Badiali \\ LECA, ENSCP-Université Pierre et Marie Curie, \\ 4 Place Jussieu, 75230 Paris Cedex 05, France
}

Received July 18, 2005

\begin{abstract}
The Boltzmann expression for entropy represents the traditional link between thermodynamics and statistical mechanics. New theoretical developments like the Unruh effect or the black hole theory suggest a new definition of entropy. In this paper we consider the thermodynamics of black holes as seriously founded and we try to see what we can learn from it in the case of ordinary systems for which a pre-relativistic description is sufficient. We introduce a space-time model and a new definition of entropy considering the thermal equilibrium from a dynamic point of view. Then we show that for black hole and ordinary systems we have the same relation relating a change of entropy to a change of action.
\end{abstract}

Key words: thermodynamics, entropy

PACS: 03.65.Ca, 05.30.-d, 05.70.-a, 47.53.+n

\section{Introduction}

Thermodynamics was created during the nineteenth century by engineers interested in the development of heat machines [1]. Today, thermodynamics appears as a fundamental part of science having some consequences in each domain of physics. Einstein established a clear separation between thermodynamics and other domains of investigation in physics [2]. For him, thermodynamics indicates what is impossible: the perpetuum motion of second kind. Einstein was inspired by this point of view when he created the special relativity, which tells us that the information cannot propagate more rapidly than the velocity of light.

Thermodynamics is based on two laws [1]; the first says that the energy is conserved while the second explains that not all transformations are possible. The second law invokes a quantity called the entropy, $S$. However this law is more than the existence of $S$, it expresses the time-evolution of $S$. For Planck and Einstein the law of evolution of a system is precisely this law of evolution of entropy [2].

Thermodynamics does not deal with the nature of matter. Atoms are not required to establish the laws of thermodynamics. Nevertheless, starting from a de- 
scription at a microscopic level, Boltzmann initiated a considerable improvement in understanding the entropy by its fundamental relation

$$
S=k_{\mathrm{B}} \ln W
$$

that asserts that thermodynamic entropy is related to the number of states $W$ consistent with some external constraints. The relation (1) is considered to be the fundamental equation of statistical mechanics; this is the entropy representation of statistical mechanics or its microcanonical form [3]. It is not surprising that after establishing (1), Boltzmann tried to describe the temporal evolution of $S$ via the existence of the so-called "H-theorem" [4].

Today the relation between thermodynamics and statistical mechanics represented by (1) is challenged by two kinds of results caused by the relativity theory. The first is related with the so-called thermodynamics of black holes [5]. Since this thermodynamics looks like the standard one we have to answer the first question: what is the connection between Einstein equations and thermodynamics. One suggestion is that the space-time geometry is needed in order to have thermodynamics: the Einstein equations have been derived from the conditions of the existence of thermodynamics near the black hole [6] or near the horizon [7]. The second question relates to the physical origin of the black hole entropy. It is implicitly assumed that this entropy must be explained starting from (1). However after thirty years of intensive work in this direction we have still to deal with questions related to the nature and the location in space of the microstates involved in $W[8,9]$. The second challenge associated with (1) is even more drastic; it is connected with the so-called Unruh effect (see [10]) which shows that the number of microstates of a unique system can be different from the two observers in uniformly accelerated motion of one relatively to the other. This effect results from the fact that we cannot define a unique quantum vacuum for these two observers.

The results mentioned above suggest that a new definition of entropy more general that the one introduced by Boltzmann might exist [11]. Of course, there is no need to search for such a new definition of entropy if we concentrate our investigations on simple systems for which a pre-relativistic description is sufficient. Nevertheless from the point of view of unification of physics it should be interesting to treat all the systems on the same or at least similar grounds. This paper is an illustration showing that some general results obtained in the domain of black hole can be extended to ordinary systems producing an improvement in our general understanding of thermodynamics. In this respect, the usual problem of black hole theory turns out to be inverted.

Hereafter we focus on one aspect of the black hole theory: the existence of a relation between the gravitational action and the space-time free energy leading to a relation between such an action and entropy. Since this result seems firmly established it seems natural to ask whether a similar relation might exist in the case of ordinary systems. It is noteworthy that the existence of a relation between action and entropy has a long history starting with Boltzmann and Poincaré prolongated by Eddington a long time ago and reinvestigated later by de Broglie (for a review 
in this domain see [12]). Both Eddington and de Broglie were searching a relation between two quantities that are considered to be relativistic invariants in restricted relativity.

To establish a relation between action and entropy we need a deep change in our traditional point of view in two different aspects. First, the action is a dynamical quantity and its calculation requires an explicit introduction of a time interval. Therefore, in order to link action and entropy means that we must have a dynamical description of the thermal equilibrium. In particular, this means that we cannot start with the description in terms of Gibbs ensemble. Thus we must conclude that, at least in some cases, entropy is not necessarily related to the counting of microstates. Second, if we want to establish a relation between action and entropy similar to the one existing in black hole physics we have to adopt a space-time point of view. A space-time model has already been introduced in recent papers and much interest associated with such a description has been shown $[13,14]$.

In summary, it should be interesting to introduce a definition of entropy without any reference to the counting of microstates but which is capable of reproducing the classical results based on (1) in the case of ordinary systems and which is also similar to the form used in describing the black hole thermodynamics.

The paper is organized as follows. In section 2 we briefly summarized the relation between action and entropy in the case of black holes. Then in section 3 we briefly describe a space-time model leading to dynamical version of thermodynamic equilibrium. In section 4 we derive the relation between action and entropy focusing on the case of ordinary systems. A discussion of the results is presented in the last section.

\section{Relation between entropy and action in the case of black holes}

After investigating the mechanical properties of a black hole [15] and the introduction of the temperature $T_{\mathrm{H}}$ via the discovery of the Hawking radiation [16] it became clear that the so-called thermodynamics of black hole has a deep meaning and looks like the usual thermodynamics provided a generalized second law is introduced [11]. Moreover, we can note that the results have been established in the standard spirit of thermodynamics [1]. We ignore what is inside the black hole as well as any microscopic description but we essentially focus on the exchange between black hole and its environment.

Later, Gibbons and Hawking [17] observed that it is possible to deduce thermodynamics of black hole from statistical mechanics i.e. from a partition function. The main steps in their derivation are as follows. A canonical partition function, $Z_{\mathrm{GH}}$, is introduced using a path integral

$$
Z_{\mathrm{GH}}=\int \mathcal{D}[g] \exp -\frac{1}{\hbar} A^{\mathrm{E}}[g]
$$

in which $A^{\mathrm{E}}[g]$ is the Euclidean action of the gravitational field associated with 
the metric $g$, and $\mathcal{D}[g]$ means that we have to perform a functional integration over all the possible metrics. In (2) the action is defined on a time interval $\beta \hbar=$ $\hbar /\left(k_{\mathrm{B}} T_{\mathrm{H}}\right)$. When a zero loop approximation is performed we get a free energy, $F_{\mathrm{GH}}$, given by $\beta F_{\mathrm{GH}}=-\ln Z_{\mathrm{GH}}=\hbar^{-1} A^{\mathrm{E}}$ where $A^{\mathrm{E}}$ is the Euclidean action calculated with the standard metric. From the usual thermodynamic relation it is possible to calculate the entropy. For four different metrics, it has been shown that the entropy derived in this way agrees with the one obtained from thermodynamic analysis. Thus the thermodynamic entropy coincides with the one derived from the spacetime properties, which is not based on an explicit counting of microstates.

For a Schwartschild black hole having an area $A$ we have $A^{\mathrm{E}}=-A c^{3}(4 G)^{-1}$ where $c$ and $G$ are respectively the velocity of light and the gravitational constant [5]. The entropy is given by $S=k_{\mathrm{B}} c^{3}(4 G \hbar)^{-1} A$ leading to the relation $S / k_{\mathrm{B}}=-A^{\mathrm{E}} / \hbar$. In the spirit of standard thermodynamics we may write this result according to

$$
\frac{\delta S}{k_{\mathrm{B}}}=-\frac{\delta A^{\mathrm{E}}}{\hbar}
$$

showing that a change in the Euclidean action produces a change in the thermodynamic entropy.

\section{Space-time model}

For pre-relativistic systems we may introduce a space-time model. This one has been already presented in [13] and [14]. Therefore, hereafter, we only point out the physical ingredients on which it is built up. We assume that the space-time points $\left(t_{i}, x_{i}\right)$ are located on the sites of a regular lattice on which there exists a relation between the elementary length $\Delta x$ and elementary time interval $\Delta t$. In the presence of a mass, $m$, we assume that $(\Delta x)^{2} / \Delta t=\hbar / m$, a relation that is sufficient to mimic a sort of Heisenberg uncertainty relations. We assume that the free motion is as simple as possible. By definition, the path associated with a given motion corresponds to a set of sites $\left(t_{i}, x_{i}\right)$; the values of $t_{i}$ are such as $t_{i+1}>t_{i}$ whatever $i$ and the coordinate positions, $x_{i+1}$ is necessarily one of the nearest neighbours of $x_{i}$, thus a path corresponds to a random walk. Thus, in this space-time model we have a discrete manifold, a quantification via the relation between $\Delta x$ and $\Delta t$ and the kinematics is defined in terms of paths on which there exists a causal relation. Our approach is reminiscent of the causal sets theory $[18,19]$. The causal relation does not fix a metric even with the relation between $\Delta x$ and $\Delta t$. As in the causal sets theory to define a length we have to say, for instance, how many space-time points exist in a given volume. This can be done if, from a physical argument, we may fix the value of $\Delta x$, for instance.

In the absence of such cutoff the limits $\Delta x, \Delta t \rightarrow 0$ taken with the constraint $(\Delta x)^{2} / \Delta t=\hbar / m$ lead to a continuous diffusion process [20] for which the diffusion coefficient is $D=\hbar / 2 m$. In the presence of an external potential, $u(t, x)$, this one is simply added to the diffusion equation [13]. Using the Feynman-Kac formula, the fundamental solution, $q\left(t_{0}, x_{0} ; t, x\right)$, of this new equation appears as a weighted sum 
of all the paths connecting the space-time points $\left(x_{0}, t_{0}\right)$ to $(x, t)$; we have

$$
q\left(t_{0}, x_{0} ; t, x\right)=\int \mathcal{D} x(t) \exp -\frac{1}{\hbar} A^{\mathrm{E}}\left[x(t) ; t, t_{0}\right],
$$

where $\mathcal{D} x(t)$ means the measure for the functional integral and

$$
A^{\mathrm{E}}\left[x(t) ; t, t_{0}\right]=\int_{t_{0}}^{t}\left[\frac{1}{2} m\left[\frac{\mathrm{d} x\left(t^{\prime}\right)}{\mathrm{d} t^{\prime}}\right]^{2}+u\left(t^{\prime}, x\left(t^{\prime}\right)\right)\right] \mathrm{d} t^{\prime} .
$$

At this level it is important to underline several points. The paths are associated with processes that occur in real time. These processes are generated from the Euclidean action $A^{\mathrm{E}}\left[x(t) ; t, t_{0}\right]$ and they are such that, on the average, there is no derivative i.e. no velocity in the usual sense on the paths [21]. The continuous limits $\Delta x, \Delta t \rightarrow 0$ are useful to give a physical meaning to the processes but, at least in principle, the explicit calculation of the path integral can be performed with finite values of $\Delta x$ and $\Delta t$ that appear as natural cutoffs in the discretization procedure needed to calculate (4). The function $q\left(t_{0}, x_{0} ; t, x\right)$ is not a density of probability although it verifies a Chapman-Kolmogorov law of composition.

To define the order - or disorder - in space-time we adopt a local definition. Around a point $x_{0}$ we count the number of closed paths that we can form during a given time interval $\tau$. If there is only one possible path we can say that we have a perfect order, no fluctuation around this path is accepted. However, after introducing a given measure, some fluctuations can take place and we have to deal with a given number of acceptable paths. For this measure, we associate the order in spacetime with this number of paths. The total order in our system will be obtained by summing the result of this procedure on all the points $x_{i}$ existing in space. This definition seems quite natural anytime we have to deal with the processes occurring in a given space-time. Of course such a definition is not unique but it is probably the simplest one.

By analogy with the thermodynamic entropy, which is defined for given values of internal energy and volume we consider that our spacetime system is prepared with a given energy $U$ and fills a volume $V$. We define a path-entropy by counting the number of closed paths for which the Euclidean action that we note hereafter as $A^{\mathrm{E}}[x(t) ; \tau]$, does not deviate too much from the action $\tau U$. In reference to the standard thermodynamics we define a path-entropy, $S_{\text {path }}$, according to

$$
S_{\text {path }}=k_{\mathrm{B}} \ln \int \mathrm{d} x_{0} \int \mathcal{D} x(t) \exp -\frac{1}{\hbar}\left[A^{\mathrm{E}}[x(t) ; \tau]-\tau U\right] .
$$

$S_{\text {path }}$ can be also rewritten as

$$
S_{\text {path }}=\frac{k_{\mathrm{B}} \tau}{\hbar} U+k_{\mathrm{B}} \ln Z_{\text {path }}
$$

with

$$
Z_{\text {path }}=\int \mathrm{d} x_{0} \int \mathcal{D} x(t) \exp -\frac{1}{\hbar} A^{\mathrm{E}}[x(t) ; \tau]=\int \mathrm{d} x_{0} q\left(0, x_{0} ; \tau, x_{0}\right)
$$


in which $q\left(0, x_{0} ; \tau, x_{0}\right)$ corresponds to closed paths for which $t_{0}=0$ and the time interval $\tau$. $Z_{\text {path }}$ is the total number of closed paths that we may count during $\tau$ irrespective of the value of $U$. $S_{\text {path }}$ contains two external parameters $\tau$ and $U$ while $Z_{\text {path }}$ is only the function of $\tau$. We may characterize the dependence of $S_{\text {path }}$ versus these two parameters by considering the two derivatives : $\mathrm{d} S_{\text {path }} / \mathrm{d} U$ defined as $T_{\text {path }}{ }^{-1}$ and $\mathrm{d} S_{\text {path }} / \mathrm{d} \tau$. From the results given in [13] we have :

$$
\frac{\hbar}{k_{\mathrm{B}}} \frac{\mathrm{d} S_{\mathrm{path}}}{\mathrm{d} U}=\frac{\hbar}{k_{\mathrm{B}}} \frac{1}{T_{\text {path }}}=\tau+\left[U-\left(\left\langle u_{\mathrm{K}}\right\rangle_{\text {path }}+\left\langle u_{\mathrm{P}}\right\rangle_{\mathrm{path}}\right)\right] \frac{\mathrm{d} \tau}{\mathrm{d} U}
$$

and

$$
\frac{\hbar}{k_{\mathrm{B}}} \frac{\mathrm{d} S_{\mathrm{path}}}{\mathrm{d} \tau}=\left[U-\left(\left\langle u_{\mathrm{K}}\right\rangle_{\mathrm{path}}+\left\langle u_{\mathrm{P}}\right\rangle_{\mathrm{path}}\right)\right]+\tau \frac{\mathrm{d} U}{\mathrm{~d} \tau}
$$

in which a relation between $U$ and $\tau$ is assumed. The averages over paths that appear in (9) and (10) are defined according to

$$
\left\langle u_{\mathrm{P}}\right\rangle_{\mathrm{path}}=\frac{1}{Z_{\mathrm{path}}} \int \mathrm{d} x_{0} u\left(x_{0}\right) q\left(0, x_{0} ; \tau, x_{0}\right)
$$

and

$$
\frac{m}{2}\left\langle\left(\frac{\delta x}{\delta t}\right)^{2}\right\rangle_{\text {path }}=\frac{\hbar}{2 \delta t}-\left\langle u_{\mathrm{K}}\right\rangle_{\mathrm{path}}
$$

in which $\left\langle u_{\mathrm{K}}\right\rangle_{\text {path }}$ is a well behaved function in the limit $\delta t \rightarrow 0$. Moreover we have checked on the examples that $\left\langle u_{\mathrm{K}}\right\rangle_{\text {path }}$ is just the usual thermal kinetic energy [13].

The quantities, $S_{\text {path }}, Z_{\text {path }}, T_{\text {path }}$ and $\mathrm{d} S_{\text {path }} / \mathrm{d} \tau$ are well defined. They give us a global characteristic of the spacetime structure but none of them corresponds to a thermodynamic quantity.

In (9) the sum $\left\langle u_{\mathrm{K}}\right\rangle_{\text {path }}+\left\langle u_{\mathrm{P}}\right\rangle_{\text {path }}$ is only dependent on the parameter $\tau$ and we may choose it in such a way that the previous sum coincides with $U$, thus $\tau$ corresponds to a condition of thermal equilibrium. Now from (9) we conclude that the relation between $\tau$ and the temperature $T_{\text {path }}$ is $\tau=\hbar /\left(k_{\mathrm{B}} T_{\text {path }}\right)$ whatever the value of $\mathrm{d} \tau / \mathrm{d} U$. If we identify $T_{\text {path }}$ with the usual temperature we can see that $Z_{\text {path }}$ defined in (8) becomes identical to the traditional partition function expressed in terms of path integral [22] and thus we may recover all the results of thermodynamics. The meaning of $\tau$ has been analyzed in detail in $[13,14]$. The relation $\tau=\beta \hbar$ is extensively used in the derivation of the black hole properties, which will be discussed below in this context. Here, to get the value of $\tau$ we do not need the Schrödinger equation or the canonical form of the density matrix. More generally what we need to derive from statistical mechanics is the existence of relations that mimic the Heisenberg uncertainty relations and define the spacetime fine structure.

\section{Relation between action and entropy in the case of ordinary systems}

In the previous section we have developed a dynamical approach to the thermodynamic equilibrium in which a time interval $\tau$ is associated with the reverse of the tem- 
perature. We have shown that the free energy defined according to $F=-k_{\mathrm{B}} T \ln Z$ is a functional of an Euclidean action $A^{\mathrm{E}}\left[x(t) ; t, t_{0}\right]$ as in the case of black hole. Before setting up a relation between action and entropy we analyze the relation between the lagrangian and Euclidean version of the action.

In (11) we have defined the potential energy $\left\langle u_{\mathrm{P}}\right\rangle_{\text {path }}$ by an average over the paths while the mean value of the kinetic energy over the paths will be defined by $m / 2\left\langle(\delta x / \delta t)^{2}\right\rangle_{\text {path }}$ that we have introduced in (12). As usually in mechanics we may define a lagrangian by the difference between kinetic and potential energy. For a given temperature, $T$, the average over the paths of this lagrangian, $\langle L(T)\rangle$, is given by

$$
\langle L(T)\rangle=\left[\frac{m}{2}\left\langle\left(\frac{\delta x}{\delta t}\right)^{2}\right\rangle_{\mathrm{path}}-\left\langle u_{\mathrm{P}}\right\rangle_{\mathrm{path}}\right]=\frac{\hbar}{2 \delta t}-\left[\left\langle u_{\mathrm{K}}\right\rangle_{\mathrm{path}}+\left\langle u_{\mathrm{P}}\right\rangle_{\mathrm{path}}\right]=\frac{\hbar}{2 \delta t}-U,
$$

in which we have used (12) and the last equality results from the equilibrium condition discussed in the previous section. From (13) we see that $\langle L(T)\rangle$ is ill defined if the time interval $\delta t$ on which we calculate the kinetic energy goes to zero. This is the consequence of the fractal character of the paths [21]. Instead of (13) we can consider the product $\langle A(T, \delta t)\rangle=\langle L(T)\rangle \delta t$ given by

$$
\langle A(T, \delta t)\rangle=\frac{\hbar}{2}-U \delta t
$$

In the limit $\delta t \rightarrow 0,\langle A(T, \delta t)\rangle$ represents the quantum of action, and thus the action is not a differentiable function of time as expected. We may consider $\langle A(T, \delta t)\rangle$ as the elementary lagrangian action associated with the time interval $\delta t$. In parallel, it seems normal to consider $U \delta t$ as the elementary Euclidean action $\mathcal{A}^{\mathrm{E}}(T, \delta t)$ for a time interval $\delta t$ since $U$ contains the sum of kinetic and potential energies. Thus, equation (14) gives the relation between the lagrangian and Euclidean elementary actions in our space-time model. It also shows that, for fixed $\delta t$, the change in one action is the same as the change in the other but with the opposite sign, we have $\delta(\langle A(T, \delta t)\rangle)=-\delta\left(\mathcal{A}^{\mathrm{E}}(T, \delta t)\right)$.

If we increase the temperature by $\delta T$ at a constant volume we have

$$
\langle A(T+\delta T, \delta t)\rangle-\langle A(T, \delta t)\rangle=-\delta t[U(T+\delta T)-U(T)]=-\delta t T \delta S
$$

in which the last equality is a consequence of the usual thermodynamic relation applied to $U$, which is also the internal energy of the system [13]. The net change of action, $\delta A_{\text {path }}$, on the time interval $\tau$ will be obtained by summing up $[\langle A(T+$ $\delta T, \delta t)\rangle-\langle A(T, \delta t)\rangle]$ on all the elementary time interval covering the total time interval $\tau$. For the last member of the equation (15) this will be simply done by multiplying the previous result by the number of elementary steps i.e. $\tau / \delta t$. It can be easily seen that the final result can be written as

$$
\frac{\delta A_{\mathrm{path}}}{\hbar}=-\frac{\delta S}{k_{\mathrm{B}}}
$$


This is the major result of this section, it shows that the change of entropy is equivalent to the change in the lagrangian action. Now we may compare this relation with (3). We have already seen above that $\delta(\langle A(T, \delta t)\rangle)=-\delta\left(\mathcal{A}^{\mathrm{E}}(T, \delta t)\right)$, leading to $\delta A_{\text {path }}=-\delta \mathcal{A}^{\mathrm{E}}$. Moreover, we have now to take into account the lack of consistency in the definition of the Euclidean actions. The action used in mechanics (like $\mathcal{A}^{\mathrm{E}}$ ) and the one used in quantum field theory (like $A^{\mathrm{E}}$ ) are defined with an opposite sign (see for instance [23]) and then we have $\delta \mathcal{A}^{\mathrm{E}}=-\delta A^{\mathrm{E}}$ from which we conclude that (16) and (3) are identical.

\section{Discussion}

In this paper we have shown that i) there exists a relation between the changes of entropy and the action and that ii) this relation is the same for ordinary systems and for the systems including a black hole. Is this a very general result having a very deep meaning?

Let us first return to the case of black hole. It is well established that a stationary black hole is actually a state of thermal equilibrium and the black hole radiates to infinity as a perfect black body at the temperature $T_{\mathrm{H}}$ [11]. Thus the meaning of $T_{\mathrm{H}}$ is strongly founded. To establish (3) we have used (2) to calculate the contribution of the metric to the partition function. The use of (2) seems a natural extension of what is done in ordinary statistical physics. However, it seems difficult to see if the introduction of $\tau$ is more than a trick connected with a continuation of time in the imaginary domain. A simple way to justify the value of $\tau$ has been proposed by Hawking starting from an analogy between the calculation of (2) and the usual methods of quantum field theory [24]. Since the Euclidean action for gravitational field and the thermal Green functions are periodic in imaginary time [25] it seems logical to identify the two periods and, furthermore, to consider that the action has to be evaluated for the time interval corresponding to this period.

In our derivation we work in real time and the time interval $\tau$ results from an equilibrium condition. The transitions in space-time are characterized by a real valued function that is in no way the solution of a time imaginary Schrödinger equation [13]. We have shown that we may derive a Schrödinger equation starting from the space-time model by forcing the system to be time-reversible. In this case, the system evolution does not require one real valued function but two real valued functions that we can mix into one complex valued function verifying a Schrödinger equation. The main point is that the potential acting in space-time is different from the one that appears in the Schrödinger equation [13]. Thus all the results that we have obtained in this paper do not need the Schrödinger equation, in a similar way it has been established that the thermodynamic relations for the black hole physics do not depend on the precise form of the Einstein equation [11].

In order to conclude we may think that the relation between entropy and action has a real meaning. For ordinary systems it represents an alternative point of view but more deeply it also shows that some results of black hole theory also exist for 
an ordinary system. Finally, since the lagrangian action is a four-dimensional scalar we see that (16) establishes a link between entropy and the relativistic invariant.

\section{References}

1. Van Ness H.C. Understanding Thermodynamics. Dover, New York, 1969.

2. Einstein A. Autobiographical Notes, Schilpp P.A. Open court Publishing Company, La Salle Illinois, 1982.

3. Callen H.B. Thermodynamics and an Introduction to Thermostatistics. Wiley, New York, 1985.

4. Boltzmann L. Lectures on gas theory. Dover, New York, 1995.

5. Ross S.F. ArXiv, hep-th, 0502195.

6. Jacobson T., Phys. Rev. Letts, 1995, 75, 1260.

7. Padmanabhan T., Class. Quant. Grav., 2002, 19, 5387.

8. Bekenstein J.D. - In: Proceedings of the seventh Marcel Grossmann Meeting, Jantzen R.T., Kaiser G.M., Ruffini R. editors, World Scientific, Singapore, 1996. ArXiv: gr-qc/9409015.

9. Sorkin R.D. ArXiv: hep-th/0504037.

10. Marolf D., Minic D., Simon S.F., Phys. Rev. D, 2004, 69, 064004; ArXiv: hep-th/0310022.

11. Wald R.M., Class. Quantum. Grav., 1999, 16, 177; Wald R.M. ArXiv: gr-qc/9702022.

12. de Broglie L. Diverses questions de mécanique et de thermodynamique classiques et relativistes. Springer Verlag, Berlin, 1986.

13. Badiali J.-P., J. Physics A, 2005, 38, 2835; ArXiv: quant-ph/0409138.

14. Badiali J.-P., Condens. Matter Phys., 2003, 6, 1.

15. Bekenstein J.D., Phys. Rev. D, 1973, 7, 2333.

16. Hawking S.W., Commun. Math. Phys., 1975, 43, 199.

17. Gibbons G.W., Hawking S.W., Phys. Rev. D, 1977, 15, 2572.

18. Bombelli L., Lee J., Mayer D., Sorkin R.D., Phys. Rev. Lett., 1987, 59, 521.

19. Rideout D.P., Sorkin R.D., Phys. Rev. D, 1999, 61, 024002.

20. Itzykson C., Drouffe J.M. Statistical Field Theory. Cambridge University Press, Cambridge, 1989.

21. Badiali J.-P., Phys. Rev. E, 1999, 60, 2533.

22. Feynman R.P., Hibbs A.R. Quantum mechanics and path integrals. Mc Graw Hill, New York, 1965, Chapter 10.

23. Le Bellac M., Barton G. Quantum and statistical field theory. BookNews, Portland, Or, USA, 1988.

24. Hawking S., Penrose R. The nature of space and time. Princeton University Press, Princeton, 1996.

25. Fulling S.A., Ruijsenaars S.N.M., Phys. Reports, 1987, 152, 135. 
Концепція ентропії. Зв'язок між дією та ентропією

\author{
Ж.-П.Бадіалі \\ Університет П'єра та Марії Кюрі, \\ пл. Жюсс'ї 4, 75230 Париж, Франція
}

Отримано 18 липня, 2005

Больцманівський вираз для ентропії представляє традиційний зв'язок між термодинамікою та статистичною механікою. Нові теоретичні результати типу ефекта Анру чи теорії чорних дір допускають нове означення ентропії. В цій статті ми розглядаємо термодинаміку чорних дір як основу і пробуємо побачити, що можна з неї отримати для випадку звичайних систем, для яких достатнім $\epsilon$ нерелятивістський опис. Ми вводимо модель простору-часу та означення ентропії, розглядаючи термічну рівновагу з динамічної точки зору. Далі ми показуємо, що для чорної діри та звичайних систем ми маємо однакове співвідношення, що пов'язує зміну ентропії зі зміною дії.

Ключові слова: термодинаміка, ентропія

PACS: 03.65.Ca, 05.30.-d, 05.70.-a, 47.53.+n 